\title{
PROTOZOAIRE CELOMIQUE
}

\section{(CELLOMOPLASMA RHIPICEPHALI N. SP.)}

TRANSMIS HÉRÉDITAIREMENT,

\section{PARASITE DE LA TIQUE RHIPICEPHALUS BURSA}

\author{
Par E. BRUMPT
}

Depuis plusieurs années, nous examinons systématiquement le contenu cœlomique des nombreux ixodinés que $\mathrm{H}$. Velu fait récolter à notre intention sur des bovidés marocains, avec l'espoir d'y rencontrer des trypanosomes, comme ceux qui ont été signalés dans diverses espèces de tiques par Leydig, O'Farrel et Machattie. Nous avons eu ainsi l'occasion de trouver un parasite nouveau.

Depuis le mois de juillet de l'année 1936, nous avons examiné un total de 876 femelles de Rhipicephalus bursa, sur lesquelles 50 présentaient le parasite cœlomique, soit dans la proportion de 5,7 pour 100.

Nous donnons ci-dessous le détail de ces divers examens et les dates de leur récolte, bien que la saison ne semble avoir aucune influence sur la fréquence des parasites.

Ce protozoaire, parfois très abondant dans le liquide cœlomique que l'on peut faire sourdre en coupant les pattes ou en piquant délicatement avec une aiguille un point quelconque de l'abdomen sans perforer un cæcum intestinal, présente la morphologie représentée dans la figure 1.

A l'état frais, ce parasite, nettement hétéropolaire, est entraîné passivement dans les courants de la préparation faite entre lame et lamelle, mais il ne semble pas présenter de mouvements actifs comme les sporozoïtes d'hémogrégarines.

Après coloration par la méthode panoptique de Pappenheim, ce germe laisse apparaitre un noyau fortement coloré en rouge violacé, entouré d'une zone claire à peine teintée qui se détache sur la masse protoplasmique rougeâtre. Cet aspect est bien différent de celui des sporozoïtes d'hémogrégarines de tiques (fig. 2), qui se colorent en bleu violacé par la même technique, et montrent une mobilité plus ou moins grande, suivant la tempéraure à laquelle ils ont été examinés.

Annales de Parasitologie, T. XVI, No $4,-1^{\text {er }}$ juillet 1938 , p. 364-368. 


\begin{tabular}{|c|c|c|c|}
\hline N"* D'EXPÉRIENCES & DATES & $\begin{array}{l}\text { NoMBRE } \\
\text { DE TIQUES } \\
\text { EXAMINÉES }\end{array}$ & $\begin{array}{l}\text { NoMBRE } \\
\text { DE TIQUES } \\
\text { INFECTÉES }\end{array}$ \\
\hline $1.326 / \mathrm{XX} \ldots \ldots \ldots \ldots \ldots$ & $2 / 7 / 36$ & 40 tiques & 0 \\
\hline $1.423 / \mathrm{XX} \ldots \ldots \ldots \ldots \ldots \ldots$ & $10 / 7 / 36$ & 34 & 5 posilives \\
\hline $38 / \mathrm{XXI} \ldots \ldots \ldots \ldots \ldots$ & $15 / 7 / 36$ & 15 & 0 \\
\hline $112 /$ XXI $\ldots \ldots \ldots \ldots \ldots$ & $22 / 7 / 36$ & 12 & 0 \\
\hline $1.089 / \mathrm{XX} 1 \mathrm{I} \ldots \ldots \ldots \ldots \ldots$ & $10 / 5 / 37$ & $56-$ & 0 \\
\hline $1.125 / \mathrm{XXII} \ldots \ldots \ldots \ldots \ldots$ & $11 / 5 / 37$ & 34 & 0 \\
\hline $1.156 /$ XXII .............. & $19 / 5 / 37$ & 90 & 0 \\
\hline $1.177 /$ XXII $\ldots \ldots \ldots \ldots \ldots$ & $19 / 5 / 37$ & 50 & 0 \\
\hline $31 /$ XXIII.............. & $25 / 5 / 37$ & 70 & 9 positives \\
\hline $85 /$ XXIII.............. & $26 / 37$ & & \\
\hline $207 /$ XXIII............. & $12 / 6 / 37$ & 110 & 27 positives \\
\hline $244 /$ XXIII. . . . . . . . . & $17 / 6 / 37$ & 18 & 0 \\
\hline $362 /$ XXIII............. & $26 / 6 / 37$ & 22 & 0 \\
\hline $3 \pi 7 / X X I V \ldots \ldots \ldots \ldots \ldots$ & $23 / 2 / 38$ & 52 & 3 positives \\
\hline $378 /$ XXIV $\ldots \ldots \ldots \ldots \ldots$ & $23 / 2 / 38$ & 59 & 2 positives \\
\hline $845 /$ XXIV $\ldots \ldots \ldots \ldots \ldots$ & $29 / 4 / 38$ & 25 & \\
\hline $927 /$ XXIV $\ldots \ldots \ldots \ldots \ldots$ & $4 / 5 / 38$ & 60 & 0 \\
\hline $1.059 / \mathrm{XXIV} \ldots \ldots \ldots \ldots \ldots$ & $11 / 5 / 38$ & 28 & 0 \\
\hline $1.193 /$ XXIV $\ldots \ldots \ldots \ldots \ldots$ & $20,5 / 38$ & 18 & . 0 \\
\hline $52 / \mathrm{XXV} \ldots \ldots \ldots \ldots \ldots$ & $10 / 6 / 38$ & $39-$ & 1 positive \\
\hline $85 / X X V \ldots \ldots \ldots \ldots \ldots$ & $15 / 6 / 38$ & $44-$ & 3 positives \\
\hline Totaux............ & & 876 tiques & 50 positives \\
\hline
\end{tabular}

Ce parasite, dont les affinités sont assez incertaines, vit exclusivement dans le liquide cœlomique. En ce qui concerne son évolution, nous possédons bien peu de renseignements; chez toutes les tiques infectées, les frottis nous ont montré exclusivement des formes simples et, sur les coupes, nous n'avons pas observé d'autres éléments permettant d'admettre un développement schizogonique ou sporogonique.

Un fait que nous croyons avoir établi est la transmission héréditaire de l'infection chez les tiques. En effet, sur 52 tiques filles (Exp. 377/XXIV), provenant d'un mélange de pontes de 27 femelles infectées (1) (Exp. 207/XXIV), élevées sur un mouton, 3 ont pré-

(1) Je remercie Mlle A. Herr pour les nombreux examens de tiques qu'elle a effectués depuis plusieurs années à mon laboratoire. 
senté les parasites cœlomiques. Dans un autre élevage de 59 tiques filles de même origine, élevées sur une vache (Exp. 378/XXIV), 2 seulement étaient infectées. Ajoutons cependant qu'il est difficile

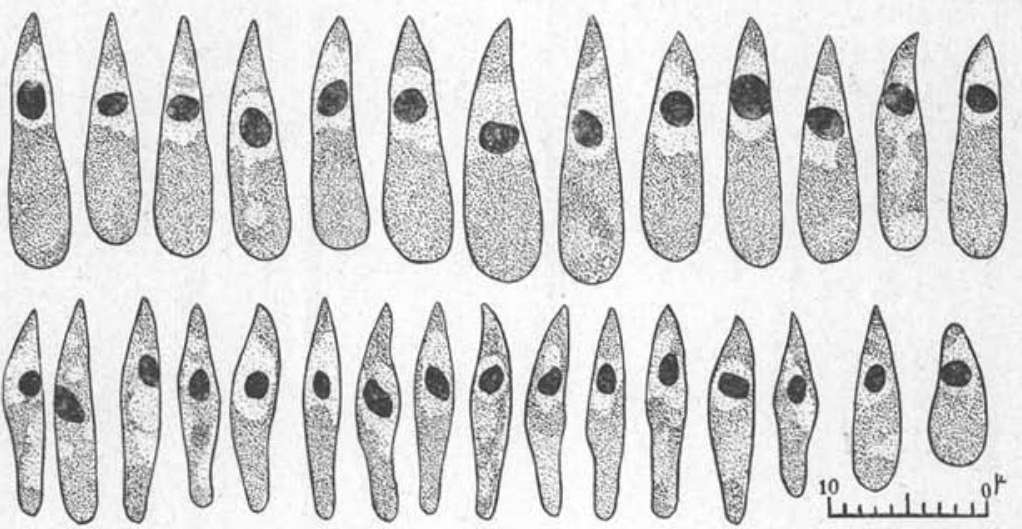

Fig. 1. - Parasite cœlomique de la tique Rhipicephalus bursa.
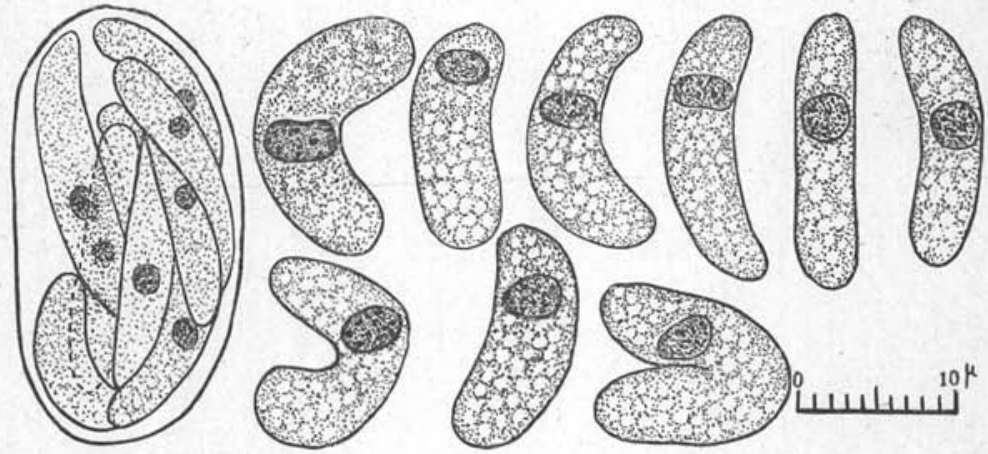

FIG. 2, - Sporozoïtes d'Hemogregarina mauritanica de la tique

Hyalomma syriacum à la même échelle que les parasites de la fig. 1 .

d'exclure la possibilité de l'infection de ces cinq tiques soit sur le mouton, soit sur le bœuf, bien que ces deux animaux, dont le sang a été examiné à de multiples reprises pour la recherche de piroplasmes et d'anaplasmes, n'aient jamais présenté aucune hémogrégarine ou quelque autre protozoaire rappelant les parasites cœlomiques des tiques. 
D'ailleurs, ces derniers, qui ne se rencontrent pas dans les glandes salivaires des rhipicéphales, ne peuvent être inoculés, et comme. d'autre part, nos grands mammifères n'ont pas eu l'occasion d'ingérer des tiques infectées au cours de nos expériences, il est plus vraisemblable d'admettre que l'infection de nos cinq tiques filles sus-mentionnées était héréditaire.

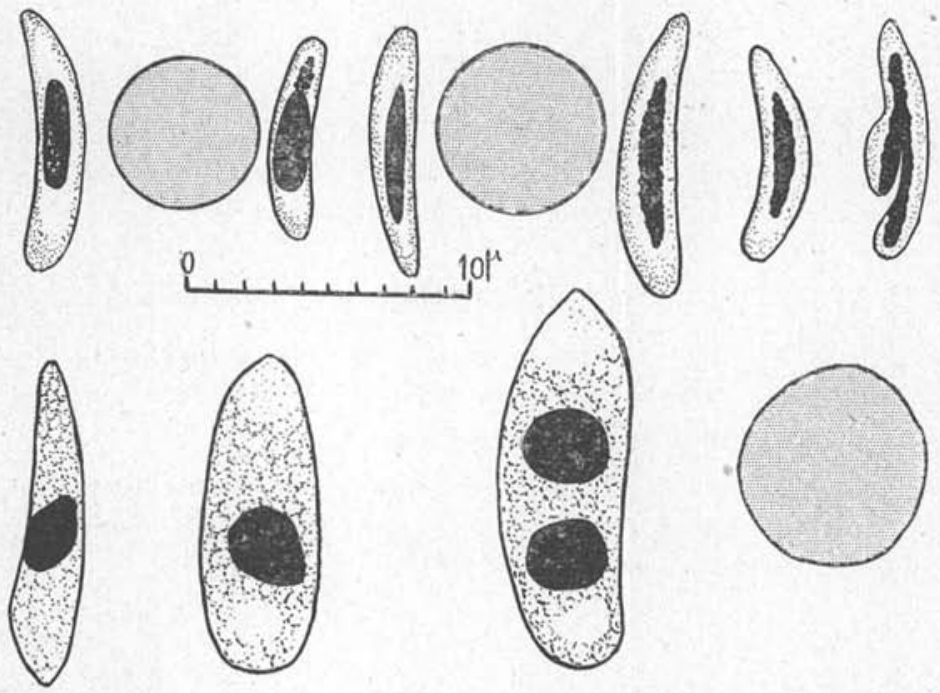

FIG. 3. - Dans la rangée supérieure, Hamogregarina bovis, d'après Martiglio et Carpano; dans la rangée inférieure, Hzmogregarina boum, d'après S. Legras.

Discussion. - Notre ignorance du cycle évolutif de ce parasite cœlomique ne nous permet pas de le classer; il ne présente ni la morphologie d'un sporozoïte, ni celle des toxoplasmes.

Il est encore plus difficile de savoir s'il appartient au cycle de l'Hæmogregarina bovis, de Martiglio et Carpano (1906) ou de l'Hæmogregarina boum, décrite par Mlle Legras (1918). Nous reproduisons, dans la figure 3 , les dessins de ces auteurs.

Dans le cas de l'H. bovis, nous admettons, comme Wenyon, qu'il s'agit probablement d'une impureté ayant contaminé les frottis, car les éléments de Martoglio et Carpano ne possèdent aucun des caractères des hémogrégarines; en revanche, nous pensons que les éléments décrits par Mlle Legras sont bien des parasites, mais nous ne croyons pas qu'il s'agisse d'hémogrégarines.

Ces protozoaires ne déterminent aucune maladie chez les tiques 
infectées, qu'il est impossible de reconnaître parmi les exemplaires indemnes, et la ponte s'effectue normalement comme chez les témoins.

L'inoculation de ces parasites (Exp. 173/XXV du 18-6-1938), dans le péritoine et sur la peau de deux spermophiles (Citillus citillus) de souris, n'a déterminé aucune infection. Nous nommons provisoirement ce parasite Coelomoplasma rhipicephali n. sp.

\section{RÉSUMÉ}

1. - Un nouveau parasite cœlomique de la tique Rhipicephalus bursa a été trouvé 50 fois sur 876 tiques examinées, soit dans 5,7 pour 100 des cas.

2. - Aucune forme de multiplication n'a été observée.

3. - Ce parasite a été transmis par la voie héréditaire 5 fois sur 111 tiques filles, soit dans une proportion sensiblement égale à celle observée dans la nature.

4. - La présence de 27 femelles infectées, sur un lot de 100 animaux, soit une proportion de 24,5 pour 100 , peut faire admettre une infection contractée sur des bœufs.

\section{Bibliographie}

Legras (Suzanne). - Sur des formes hémogrégariniennes vues dans le sang et dans le tissu ganglionnaire des bovidés d'Algéric. Bull. Soc. Path. Exot., XI, 1918, p. 274.

martoglio (F.) et Carpano (M.). - Sopra un caso di Hamogregatina bovis. Ann. Igiene sperimentale, XVI, 1906, p. 251.

Institut de parasitologie de la Faculté de médecine de Paris (Directeur : Prof. E. Brumpt). 